\title{
Aspectos quirúrgicos del cáncer de vesícula biliar
}

\author{
Fernando Maluenda G ${ }^{1,3}$, Juan C D íaz J', \\ Xabier de Aretxabala U 1,3, Patricio Burdiles $\mathrm{P}^{1}$, \\ Attila Csendes J ${ }^{1}$, Luis Contreras $M^{2}$.

\section{Strategies for the surgical treatment of gallbladder cancer}

There is controversy in some aspects of the surgical treatment of non-mucosal gallbladder carcinoma. An accurate staging based on T (wall) involvement is crucial, otherwise understanding may yield falsely pessimistic results. The decision about the type of resection to be performed should be based on patient status (age, performance, comorbidities, etc) and tumor characteristics (histological type, vascular, neural or lymphatic spread, cell differentiation, tumor involvement of surgical margins in cystic duct, etc). For muscular (T1b) involvement, there is a great controversy about performing a simple cholecystectomy or en-block radical resection. For T2 there is consensus that an en-block radical surgery including liver resection $(\mathrm{IVb}-\mathrm{V})$ and lymphonodal clearance should be performed, since this approach has a great impact in survival. The role of surgical excision for tumors with serosal or liver involvement is controversial, due to the poor survival of these patients. However we have observed a $13 \%$ actuarial survival at 5 years, in this subset of patients (Rev Méd Chile 2005; 133: 723-8).

(Key Words: Cholelithiasis; Gallbladder neoplasms, Neoplasm staging)

Recibido el 26 de julio, 2004. Aceptado el 22 de noviembre, 2004.

Departamentos de Cirugía $^{1}$ y Anatomía Patológica ${ }^{2}$, Hospital Clínico de la Universidad de Chile. Santiago de Chile.

${ }^{3}$ Departamento de Cirugía. Clínica Alemana de Santiago, Chile.

\begin{abstract}
A pesar de que el cáncer de vesícula biliar es la primera causa de muerte por cáncer en mujeres en Chile y con tasas en aumento sostenido ${ }^{1-3}$, exhibe, como pocas patologías, notoria controversia en sus aspectos terapéuticos quirúrgicos y resulta difícil plantear conductas quirúrgicas de consenso o basadas en estudios que no merezcan dudas metodológicas y que por lo
\end{abstract}

Correspondencia a: Dr. Fernando Maluenda G. Departamento de Cirugía, Hospital Clínico Universidad de Chile. Santos Dumont 999, Santiago, Chile. Fono: 026788329. Fax: 02 7327245. E mail: fmaluend@ns.hospital.uchile.cl tanto, sus resultados sean indiscutiblemente válidos. En esta controversia, son varios los factores que influyen, a saber:

a) Existe una alta prevalencia de la enfermedad en países como el nuestro, donde la investigación no está sistematizada, tiene escasos recursos materiales y tecnológicos y, salvo loables esfuerzos de algunos grupos, no existe un caudal de investigación que aporte un flujo continuo de información. b) La baja frecuencia de esta enfermedad en países del hemisferio norte, donde están los recursos materiales y humanos de dedicación exclusiva a la investigación, hace que se trate de sacar conclusiones sobre casuísticas 
retrospectivas, pequeñas, reunidas a lo largo de muchos años, en grupos cooperativos o multicéntricos con toda la variabilidad y pérdida de información que ello conlleva. c) Los estudios que existen acerca del tratamiento quirúrgico de la enfermedad, fallan en el origen de la información al no quedar claramente descrito bajo qué criterios se seleccionaron los grupos de pacientes sometidos a distintas terapias quirúrgicas, lo que determina que la conclusión resultante de la comparación de grupos, tenga un valor limitado. d) No parece factible, desde el punto de vista ético, tener estudios prospectivos randomizados para determinar el efecto de las distintas terapias resectivas en este cáncer. e) Existen claramente dos tendencias en la cirugía del cáncer vesicular: los grupos japoneses que comunican las mejores sobrevidas realizando cirugías más radicales, extensas o agresivas y los grupos norteamericanos y nacionales que muestran resultados no tan optimistas, con cirugías en general más conservadoras.

De todo esto resulta que podemos obtener ciertas sugerencias terapéuticas o conclusiones de valor limitado, que probablemente no pudieran sobrepasar estrictos criterios de rigurosidad científica.

Se da la paradoja que, mientras más precoz hacemos el diagnóstico con relación a la operación, más avanzada está en general la enfermedad. En nuestra experiencia, en solo $29 \%$ de los pacientes hacemos el diagnóstico en forma postoperatoria, postcolecistectomía, en el estudio histopatológico de la vesícula. Esta realidad determina lo importante que es para los tumores que infiltran hasta la serosa, el examen macroscópico y el estudio seriado microscópico, para precisar exactamente el grado de infiltración tumoral en la pared vesicular y excluir así las posibilidades de emor. La subclasificación conducirá a resultados falsamente pesimistas.

Etapificación. No existe un sistema de clasificación universal. Aún se hace referencia a la clasificación de Nevin $^{4}$ y se le sugieren modificaciones, sin embargo el sistema más difundido es la clasificación TNM de la Unión Internacional contra el Cáncer ${ }^{5}$. Nosotros hemos usado la versión de 1997 para describir las opciones terapéuticas y presentar nuestros resultados. La versión revisada ${ }^{6}$ de 2002 presenta cambios en la clasificación del tumor primario (T) y simplifica la infiltración linfonodal (N) como positiva o negativa, sin importar la topografía de los linfonodos comprometidos. Para poder precisar $\mathrm{N}$ se requiere la extirpación de al menos 3 linfonodos. La experiencia nacional ${ }^{7,8}$ comunica la linfadenectomía de 10 linfonodos en promedio por paciente. Informar resultados sobre la base de estadios de la clasificación TNM induce a error, de momento que la composición dentro de cada estadio tiene tumores con distinto grado de penetración. Por ello resulta muy apropiado referirse a los grupos de pacientes, agrupándolos según la infiltración del tumor primario $(\mathrm{T})$, que es un dato que siempre existe.

Procedimientos en cirugía radical. La resección quirúrgica constituye la única alternativa terapéutica potencialmente curativa para esta enfermedad. La cirugía radical en bloque tiene como objetivo extirpar las estructuras anatómicas que tienen relaciones de vecindad con la vesícula biliar, o son sus sitios de drenaje venoso, linfático o biliar y cuya resección es factible dejando márgenes libres de infiltración tumoral. Los componentes fundamentales de esta intervención son:

Hepatectomía: la magnitud de la hepatectomía va desde la resección de lecho hepático, definido como la resección de un margen de $2 \mathrm{~cm}$ de parénquima en el lecho vesicular, hasta la hepatectomía derecha extendida, pasando por subsegmentectomía IVb-V y segmentectomía IVb-V-VI. No parece haber relación directa entre la magnitud de la resección hepática y la sobrevida.

Linfadenectomía: corresponde a la extirpación de todo el tejido linfograso en los siguientes temitonios: cístico, pericoledocianos, hiliares, peripancreáticos (sólo cabeza), periduodenales, periportales, celíaco, mesentérica superior. Se debe extirpar al menos tres linfonodos para etapificar $\mathrm{N}$, siendo 10 el número promedio de linfonodos extirpados, en la experiencia nacional ${ }^{7,8}$.

Extirpación de otros órganos: se debe identificar y volver a resecar el muñón cístico para estudio biópsico contemporáneo y si resulta positivo para infiltración tumoral, procede agregar la resección de la vía biliar. Hay grupos que proponen la resección de la vía biliar de rutina en $\mathrm{T} 2^{9}$ y la duodenopancreatectomía para casos de infiltración duodenal. La resección segmentaria de colon trans- 
verso por infiltración directa es también factible. En los casos en que el diagnóstico se hizo postcolecistectomía (cáncer inaparente) es necesario agregar la resección de los sitios de entrada de los trócares de laparoscopia y del ligamento redondo, por la posibilidad de implantación en estos sitios ${ }^{10}$. En todo caso parece tener mayor utilidad como etapificación que como procedimiento terapéutico ${ }^{11}$.

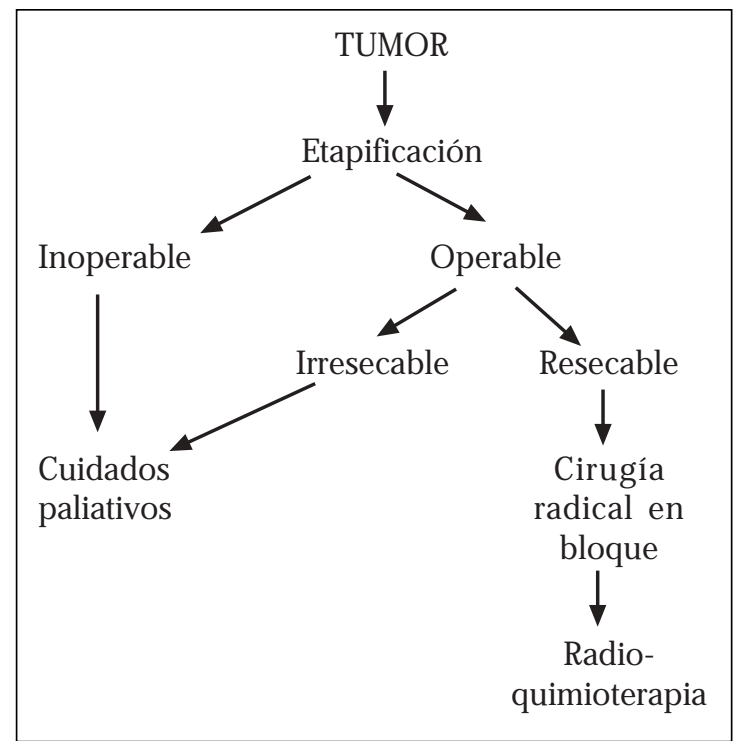

FiguRA 1. Diagnóstico preoperatorio.

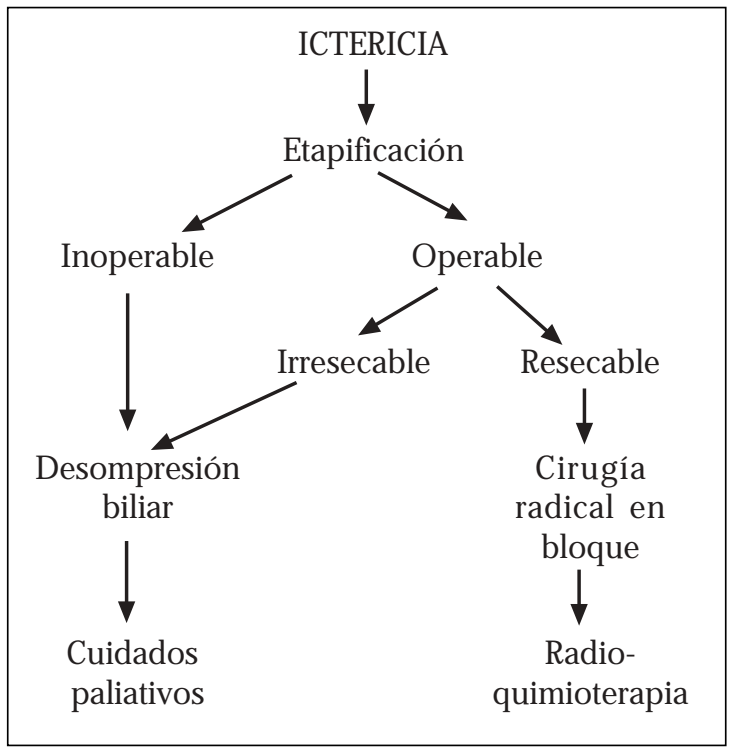

FigURA 2. Diagnóstico preoperatorio.
Qué tipo de resección aplicar a cada paciente. Hasta ahora, nos hemos basado sólo en el grado de infiltración en la pared vesicular $(\mathrm{T})$, para decidir el tipo y magnitud de la resección quirúrgica, sin embargo desde un punto de vista práctico, se debe considerar también el momento del diagnóstico en relación a la intervención quirúrgica. Otros factores, además, que debieran tomarse en cuenta son la edad del paciente y grado de diferenciación tumoral. Los pacientes menores de 40 años y los portadores de tumores indiferenciados tienen un peor pronóstico ${ }^{12}$.

A. Momento del diagnóstico. a. Preoperatorio: En $10 \%$ de nuestra casuística ${ }^{8}$ la forma de presentación es como tumor de hipocondrio derecho (Figura 1) detectado a ecotomografía abdominal o al examen físico. Se debe proceder a etapificar en base a tomografía axial computada de abdomen, radiografía de tórax y exámenes plasmáticos y hematológicos. Según éstos y la condición física del paciente, considerar la operabilidad. Si el paciente es operable, es decir, si está en condiciones generales de ser sometido a una exploración quinúrgica y desde el punto de vista oncológico no hay evidencias de metástasis regionales o a distancia, se le somete a exploración quinúrgica. Si es factible resecar el tumor con márgenes negativos microscópicos (Ro), procede la resección. De no poder lograr una resección $\mathrm{Ro}$, se debe proceder a la toma de biopsias y derivar al paciente para tratamiento quimioterápico paliativo o a unidad de cuidados paliativos. Si se logra una extirpación con criterio oncológico, a continuación se debe aplicar protocolo de radio-quimioterapia.

Una segunda forma de presentación es la ictericia, en general, sin dolor abdominal (Figura 2). En la evaluación preoperatoria se debe incluir algún método de imagen dirigido a visualizar la vía biliar y precisar el sitio y causa de la obstrucción. Si no se asocia a tumor visible, preferimos usar la colangiografía por resonancia nuclear magnética (CRNM). En caso contrario, cuando a la ictericia se le asocia un gran tumor visible, empleamos la colangiografía endoscópica retrógrada e instalación de un stent endoscópico dada la alta probabilidad de tratarse de un cáncer irresecable. De esta forma solo indicamos tratamiento paliativo endoscópico para su ictericia y el prurito asociado. Si la CRNM muestra infiltración segmentaria de la vía biliar y un adecuado margen libre de tumor en la vía biliar 
proximal, resecamos la vía biliar, practicándose una derivación biliodigestiva (hepático o colangioyeyunoanastomosis), previa realización de biopsia contemporánea del margen de sección en vía biliar. b. Intraoperatorio: la gran mayoría de los pacientes que se diagnostican en este momento corresponden a portadores de cánceres diseminados al peritoneo y el acto quirúrgico se limita a certificar el diagnóstico mediante la toma de biopsias. $\mathrm{Si}$ como hallazgo se encuentra un tumor vesicular localizado, resecable y el equipo está capacitado para efectuar cirugía radical, procede entonces la resección, previa certificación con biopsias, sin poner énfasis en el grado de penetración parietal por el tumor, dado que excluimos de la resección radical sólo a los pacientes portadores de un cáncer mucoso, que en general no se va a manifestar como tumor en el intraoperatorio (Figura 3). c. Postoperatorio: corresponde al diagnóstico hecho en el examen histopatológico de la pieza de colecistectomía por colelitiasis, momento en que son diagnosticados la mayoría de los cánceres de vesícula biliar con opciones de curación postratamiento. El lapso promedio de tiempo entre la colecistectomía y la reintervención, ha sido 32 días en nuestra experiencia. El tratamiento para los tumores diagnosticados en este período se basa en el grado de infiltración tumoral parietal.

B. Grado de infiltración tumoral (T). Basados en la clasificación TNM, la cirugía propuesta para cada nivel de infiltración, es la siguiente:

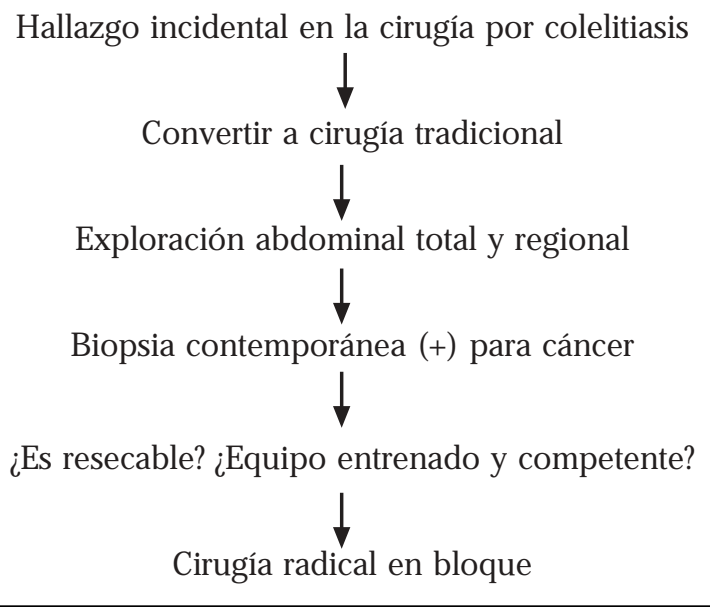

FIGURA 3. Diagnóstico intraoperatorio.
Mucoso, T1a: Existe consenso general que la colecistectomía sola es una terapia adecuada y suficiente para este tumor. Una consideración especial se debiera tener para los casos en que se haya roto la vesícula al momento de la colecistectomía y una potencial diseminación directa o por aerosolización no se pueda descartar. Cabe plantear entonces la reintervención para la resección hepática y linfadenectomía considerando además otras variables del paciente y del tumor (edad, comorbilidad, tipo histológico, diferenciación tumoral, márgen tumoral a nivel cístico, etc).

Muscular, T1b: Existe controversia acerca del tratamiento en este grupo, algunos autores ${ }^{13}$ plantean que este tumor representa una enfermedad local. Sin embargo, otros lo asumen como una enfermedad que puede diseminarse regional y sistémicamente al momento del diagnóstico. La referencia a este grupo en la literatura es escasa, con reducido número de pacientes, de 10 casos o menos. Estudios nacionales ${ }^{7}$ pioneros han desestimado la reintervención radical para este grupo dada la baja probabilidad de encontrar linfonodos positivos (0 de 10 casos) o tumor residual en hígado (1 de 10 casos). Recientemente De Aretxabala ${ }^{14}$ publicó una serie de 46 pacientes con infiltración muscular que presentaron $78 \%$ de sobrevida a 5 años, independientemente del tipo de tratamiento quirúrgico. Wakai ${ }^{15}$ comunicó una casuística de 25 pacientes y no encontró beneficio en cuanto a sobrevida ( $87 \%$ a 10 años) en el grupo sometido a cirugía radical respecto a colecistectomía simple. Para los grupos japoneses que hacen diagnóstico pre o intraoperatorio ${ }^{9}$ en alto porcentaje y por lo tanto, la decisión de cirugía radical se decide basándose en resultados de biopsia contemporánea, recomiendan la linfadenectomía de N1 por la dificultad de diferenciar entre T1 y T2 en estas condiciones, con una sobrevida de $100 \%$ a 5 años, cuando la linfadenectomía es negativa para tumor. En un estudio sobre 20 pacientes, Cubertafond ${ }^{16}$ informó sobrevida de $28 \%$ a 4 años para esta etapa, solo con colecistectomía, a pesar de que es un estudio sobre 73 centros y que podría estar incluyendo pacientes subclasificados. Shimada ${ }^{17}$ comunicó una sobrevida de todos sus casos a 5 años (n: 3 pacientes), al igual que Todoroki ${ }^{9}$ (n: 5 pacientes). Por otra parte, la serie de Ouchi ${ }^{18}$, mostró 2 pacientes fallecidos debido a recurrencia local postcolecistectomía sola y 2 pacientes libres 
de enfermedad después de haber sido sometidos a resección hepática de lecho y linfadenectomía. En nuestra casuística ${ }^{8}$ tenemos 14 pacientes con infiltración hasta la muscular, 8 de ellos aceptaron ser reintervenidos y se les realizó hepatectomía de lecho más linfadenectomía N1-N2 y 6 pacientes rechazaron la reintervención, quedándose solo con colecistectomía. Hubo N1 positivo en tres de los 8 pacientes con cirugía radical y ausencia de infiltración hepática y todos ellos están vivos con una mediana de seguimiento de 33 meses (rango 6-77). De los pacientes sometidos solo a colecistectomía, un paciente falleció por recurrencia de su enfermedad. Todas las muestras son de números pequeños para sacar cualquier conclusión. Actualmente, recomendamos en esta etapa la hepatectomía de lecho y linfadenectomía N1-N2, enfatizando en la correcta determinación del grado de penetración en la histopatología. La decisión de reintervenir debiera considerarse después de volver a revisar la pieza de colecistectomía y tomar en cuenta ciertas características propias del tumor como grado de diferenciación, presencia de invasión vascular, linfática 0 neural. Si ello no es posible, considerar la reintervención resectiva y etapificación correcta.

Subseroso, T2: Hay acuerdo general acerca de la necesidad de reintervención y resección radical en estos pacientes, dado que sería el grupo en el que mayor impacto y mejoría de la sobrevida habría. La resección hepática mínima recomendada es una hepatectomía de lecho y linfadenectomía de N1-N2. La sobrevida a 3 años con esta resección es $91 \%$, comparado con $28 \%$ después de simple colecistectomía ${ }^{19}$. Shirai ${ }^{20}$ comunicó una sobrevida a 5 años de $90 \%$ versus $40 \%$ solo con colecistectomía. De Aretxabala ${ }^{7}$ dió cuenta de sobrevida de $70 \%$ en comparación a $20 \%$ con solo colecistectomía y hay similares resultados en otro estudio reciente. No hay diferencias significativas en cuanto a sobrevida entre los pacientes sometidos a cirugía radical en un tiempo en comparación con los reoperados $7,11,21$.

Tejido adiposo: Corresponde a la infiltración de la porción de la pared vesicular situada entre la capa muscular y el hígado. Aretxabala ${ }^{7}$ ha llamado la atención sobre este grupo, enfatizando su peor pronóstico respecto de los subserosos (cara peritoneal libre), el hallazgo de tumor residual en hígado y linfonodos infiltrados es la regla en la reoperación. De 6 pacientes reintervenidos, en 3 ya había diseminación irresecable al momento de la reoperación.

Serosos con o sin infiltración hepática mayor a $2 \mathrm{~cm}$, T3-T4: El rol de la cirugía radical en este grupo es controvertido. Existen series que encuentran ausencia de sobrevida a 5 años aun con resecciones hepáticas mayores ${ }^{18,19}$. La sobrevida global a 1 año es $24 \%{ }^{19}$ y $7 \%$ a 3 años ${ }^{18}$. Estudios recientes han mostrado una sobrevida de $21 \%$ a 5 años en casos T3 y de $28 \%$ para $\mathrm{T}^{11}$ con cirugía radical. Dado que es una enfermedad de invasión fundamentalmente local, la resección en bloque con duodenopancreatectomía o hepatectomía de distinta magnitud o ambos, con resección combinada de vía biliar, es la propuesta de los grupos más agresivos $9,22,23$, tratando de lograr una resección de margen microscópico libre de tumor (Ro). Debe realizarse biopsia contemporánea de linfonodos N2 (incluyendo periaórticos). Si éstos están infiltrados, se excluye la posibilidad de una resección Ro, ya que en estos casos, la sobrevida es equivalente a la de los pacientes con metástasis a distancia ${ }^{24}$. En nuestra experiencia con pacientes T3 y resección radical, tenemos $13 \%$ de sobrevida actuarial a 5 años sobre 15 casos tratados y una mediana de 17 meses para 16 pacientes portadores de tumores T4 con sobrevida máxima de 43 meses.

Metástasis hepáticas, peritoneales o ambas: $25 \%$ de los pacientes de nuestra serie ${ }^{8}$ tenían diseminación hepática, peritoneal 0 ambas al momento del diagnóstico. No hay cabida para la cirugía en este grupo de enfermos, de ningún tipo. La mediana de sobrevida es de tres meses, habiendo tenido sobrevidas hasta de 9 meses, en pacientes que estuvieron con quimioterapia.

En suma, la cirugía resectiva del cáncer vesicular ofrece expectativas de curación para etapas tempranas, intravesiculares, en la medida que se logre una resección Ro. En etapas más avanzadas de la enfermedad se debe considerar especialmente la experiencia del equipo quirúrgico tratante. No es posible probar estadísticamente que la alternativa quirúrgica resectiva en bloque en etapas extravesiculares, es mejor que hacer nada 0 que la quimioterapia con o sin radioterapia. 


\section{REFERENCIAS}

1. Mortalidad por causa específica. Departamento estadísticas e información de salud. Ministerio de Salud. Chile, 2001.

2. Chianale J, Valdivia G, Del Pino G, Nervi F. Mortalidad por cáncer vesicular en Chile, su relación con las tasas de colecistectomía. Análisis de la última década. Rev Méd Chile 1990; 118: 1284-8.

3. Serra I, Maturana M, Medina E, Sharp A. Changing trends of gallbladder cancer in Chile. A high risk area. Int J Cancer 1990; 45: 376-7.

4. Nevin JE, Moran TJ, Kay S, King R. Carcinoma of the gallbladder. Staging, treatment and prognosis. Cancer 1976; 37:141-8.

5. Gallbladder Cancer. In: American Joint Committee On Cancer: AJCC Cancer Staging Manual. Philadelphia Pa. Lippincott-Raven Publishers. $5^{\text {th }}$ ed., 1997; 103-8.

6. Greene F, Page D, Fleming I, Fritz A, Balch C, HaLleR D, Morrow M. (Eds.). Gallbladder Cancer in: AJCC Cancer Staging Manual. Sixth Ed. Springer, 2002.

7. De Aretxabala X, Roa I, Burgos L, Araya J, Viluaseca MA, SILVA J. Curative resection in potentially resectable tumours of the gallbladder. Eur J Surg 1997; 163: 419-26.

8. Maluenda F. Cáncer de la vesícula biliar. Resultados de la cirugía resectiva. Rev Ch Cir 2002; 54: 464-73.

9. Todoroki T, KaWamoto T, TaKahashi H, Takada $\mathrm{Y}$, KoIKe N, OtsuKa M ET AL. Treatment of gallbladder cancer by radical resection. Brit J Surg 1999; 86: 622-7.

10. Fong Y, Hefrernan N, Blumgart LH. Gallbladder carcinoma discovered during laparoscopic cholecystectomy: aggressive reresection is beneficial. Cancer 1998; 83: 423-7.

11. Fong Y, JaRnagin W, BlumgarT L Gallbladder cancer: comparison of patients presenting initially for definitive operation with those presenting after prior non curative intervention. Ann Surg 2000; 232: 557-69.

12. De Aretxabala X, Roa L, Araya JC, Burgos L, Flores P, Wistuba I ET AL. Gallbladder cancer in patients less than 40 years old. Br J Surg 1994; 81: 111.
13. Shirai Y, Yoshida K, Tsukada K, Muto T, WatanaBE $H$. Radical surgery for gallbladder carcinoma. Long-term results. Ann Surg 1992; 216: 565-8.

14. De Aretxabala X, Roa I, Mora J, Pincheira O, Burgos L, Silva J et al. Cáncer de la vesícula biliar. Manejo de pacientes con invasión de la túnica muscular. Rev Méd Chile 2004; 132: 183-8.

15. WAKaI T, ShiraI $\mathrm{Y}$, Yokoyama N, NagakuRa $\mathrm{S}$, Watanabe H, HataKeyama K. Early gallbladder carcinoma does not warrant radical resection. $\mathrm{Br} \mathrm{J}$ Surg 2001; 88: 675-8.

16. Cubertafond P, Gainant A, Cucchiaro G. Surgical treatment of 724 carcinomas of the gallbladder. Results of the French Surgical Association Survey. Ann Surg 1994; 219: 275-80.

17. Shimada H, Endo I, Togo S, Nakano A, Izumi T, NAKagainara $G$. The role of lymph node dissection in the treatment of gallbladder carcinoma. Cancer 1997; 79: 892-9.

18. Ouchi K, Suzuki M, Tonninuga T, Saijo S, Matsuno S. Survival after surgery of cancer of the gallbladder. Br J Surg 1994; 81: 1655-7.

19. Yamaguchi K, Chijiwa $K$, SaiKi $S$, Nishihara $K$, TaKashima M, KaWaKami $K$ et al. Retrospective analysis of 70 operations for gallbladder carcinoma. Br J Surg 1997; 84: 200-4.

20. Shirai Y, Yoshida K, TsuKada K, Muto T. Inaparent carcinoma of the gallbladder: an appraisal of radical second operation after simple cholecystectomy. Ann Surg 1992; 215: 326-31.

21. De Aretxabala X, Roa I, Mora J, Oreliana J, Rededeman J, BuRgos L ET AL. Laparoscopic cholecystectomy: its effect on the prognosis of patients with gallbladder cancer. World J Surg 2004; 28: 544-7.

22. Bartlett Dl, Fong Y, Fortner FG, Brennan MF, BLUMGART LH. Long term results after resection for gallbladder cancer. Implications for staging and management. Ann Surg 1996; 224: 639-46.

23. Окамото A, Tsuruta $K$, Ishiwata J, Isawa $T$, Kamisawa T, TANAKA Y. Treatment of T3 and T4 carcinomas of the gallbladder. Int Surg 1996; 81: 130-5.

24. Kondo S, Nimura Y, Hayakawa N, Kamiya J, Nagino M, UESAKA K. Regional and para-aortic lymphadenectomy in radical surgery for advanced gallbladder carcinoma. Br J Surg 2000, 87: 418-22. 